\title{
Persistance d'orbites périodiques relatives dans les systèmes hamiltoniens symétriques
}

\author{
James MONTALDI \\ Institut Non Linéaire de Nice, UMR 129 CNRS-UNSA, 1361 route des Lucioles, \\ F-06560 Valbonne, FRANCE. \\ e-mail : james@doublon.unice.fr
} \begin{abstract}
système hamiltonien persiste lorsque on change de niveau d'énergie. Dans cette
Résumé. Note, on considère la question analogue pour les orbites périodiques relatives. On démontre, sous des hypothèses de non-dégénérescence de l'orbite et d'une action libre du groupe de symétries, qu'une orbite périodique relative persiste lorsqu'on change non seulement l'énergie, mais aussi la valeur de l'application moment.
\end{abstract}

Il est connu depuis Poincaré qu'une orbite périodique non-dégénérée dans un

\section{Persistence of relative periodic orbits in symmetric Hamiltonian systems}

It was known to Poincaré that a non-degenerate periodic orbit in a Hamiltonian system persists to nearby energy-levels. In this note, we consider the analogous Abstract. problem for relative periodic orbits in symmetric Hamiltonian systems. We show that non-degenerate relative periodic orbits persist to nearby values of the energymomentum map, under the hypothesis that the group of symmetries acts freely.

\begin{abstract}
Abridged English Version
Consider a symmetric Hamiltonian system, with phase space $P$, and with symmetry group a compact connected Lie group $G$, acting in a Hamiltonian fashion so that there is an equivariant momentum map $\mathbf{J}: P \longrightarrow \mathfrak{g}^{*}$, where $\mathfrak{g}$ is the Lie algebra of $G$, and $\mathfrak{g}^{*}$ its dual. We suppose throughout that the action of $G$ is free, which implies firstly that $P / G$ is a smooth manifold and secondly that the momentum map is a submersion. The orbit space is partitioned into reduced spaces $P_{\mu}:=\mathbf{J}^{-1}\left(\mathcal{O}_{\mu}\right) / G \subset P / G$, for each $\mu \in \mathfrak{g}^{*}$, where $\mathcal{O}_{\mu}$ is the coadjoint orbit containing $\mu$. Each $P_{\mu}$ is a smooth symplectic manifold, but they are not necessarily all of the same dimension.

The dynamics on $P$ are defined by a $G$-invariant Hamiltonian $H$, which defines a smooth function on $P / G$, also denoted $H$. The Poisson structure on $P / G$ induced from that on $P$ then determines the dynamics via the Hamilton-Poisson vector field $X_{H}=\{H, \cdot\}$. By conservation of momentum, the dynamics induced on $P / G$ preserves the reduced spaces.
\end{abstract}


A relative periodic orbit (or RPO) for a symmetric dynamical system is a trajectory $\gamma(t)$ for which

$$
\gamma(T)=g \cdot \gamma(0)
$$

for some $g \in G$ and some $T>0$ (the period). If we pass to the orbit space, a relative periodic orbit becomes a periodic orbit. We also denote this periodic orbit by $\gamma$. By a theorem of Field [2] and Krupa [5], a relative periodic orbit in $P$ is dense on a torus of dimension at most $\operatorname{rank}(G)+1$ (or more generally $\operatorname{rank}\left(N_{G}(K)\right)+1$ where $K$ is the isotropy group of any point of the trajectory, and $N_{G}(K)$ is the normalizer of $K$ in $\left.G\right)$.

For any non-constant periodic orbit of a hamiltonian system, the return map has eigenvalue 1 with algebraic multiplicity at least 2 , and the periodic orbit is said to be non-degenerate if this multiplicity is precisely 2. For a non-constant RPO(i.e. not a relative equilibrium) of momentum $\mu$, since momentum is preserved, we say the RPO is non-degenerate if its image in $P_{\mu} \subset P / G$ is nondegenerate in the usual sense. In the case of a genuine periodic orbit in phase space (which would often be the case in applications), the non-degeneracy condition coincides with that in $[9, \S 4(\mathrm{c})]$, namely that the Floquet multiplier 1 has algebraic multiplicity precisely $2 \operatorname{dim}(G . p)-\operatorname{dim}(G . \mu)+2$.

Theorem 1 Suppose $\gamma$ is a non-degenerate relative periodic orbit in $P_{\mu_{*}}$ of period $T_{*}$ and for which $H=E_{*}$. Then there is a neighbourhood of $\gamma$ in $P / G$ in which, for each $\mu \in \mathfrak{g}^{*}$ close to $\mu_{*}$, and each $E$ close to $E_{*}$ there is at least $1 \mathrm{RPO}$, of momentum $\mu$, of energy $E$ and of period close to $T_{*}$, and at least $w(\mu) / w\left(\mu_{*}\right)$ if they are all non-degenerate.

Here $w(\mu)$ is the Weyl number of the coadjoint orbit $\mathcal{O}_{\mu}$, which is the number of ponts in the intersection of $\mathcal{O}_{\mu}$ with a Cartan subalgebra, and also its Euler characteristic [6].

Remarks (i) The 1 or $w(\mu) / w\left(\mu_{*}\right)$ RPOs arise as fixed points of a symplectic automorphism $f_{E, \mu}$ of $\mathcal{O}_{\mu}$ which is homotopic to the identity (Lemme 2). By Lefschetz fixed point arguments, there is at least 1 fixed point, and if they are non-degenerate there are $w(\mu) / w\left(\mu_{*}\right)$. In fact, in the degenerate case, one can often do better, using the fact that $f_{E, \mu}$ is symplectic. For example, if $G=\mathbf{S O}(3)$, the coadjoint orbits are 2-spheres, and it is known that any symplectic map of the 2 -sphere has at least 2 fixed points. Furthermore, if it has more than 2 fixed points (which might be forced by further symmetry), then by a theorem of Gambaudo and Le Calvez [4] and Franks [3], it has infinitely many periodic points (of arbitrarily long period).

(ii) In applications (see $[7,11]$ ) there may be additional finite symmetry with non-trivial isotropy. Suppose the semi-direct product $G \times{ }_{s} K$ acts on $P$ and that $\gamma(t)$ has isotropy $K$ (a finite group). Then $K$ acts on the orbit space and the return map $f_{E, \mu}$ will be $K_{\mu}$-equivariant, thus possibly forcing more fixed points. See the example at the end of the paper.

Outline of proof Without loss of generality, we can consider the case $\mu_{*}=0[6, \S 2]$. Let $\bar{p}=\gamma(0)=$ G.p. In a neighbourhood $U$ of the group orbit $\bar{p}$ of zero momentum, the orbit space $P / G$ can be identified as a Poisson space with a product of a symplectic space and a Poisson space:

$$
U \stackrel{\sim}{\longrightarrow} Y \times \mathfrak{g}^{*},
$$

where $Y=\left(\operatorname{ker} d \mathbf{J}_{p}\right) / \mathfrak{g} \cdot p$ is the symplectic slice at $p[10,6]$, and can be identified with $P_{0}$ (at least in a neighbourhood of the origin). For $\mu \in \mathfrak{g}^{*}$, the reduced space $P_{\mu}$ is then identified with

$$
P_{\mu} \cap U \stackrel{\sim}{\longrightarrow} Y \times \mathcal{O}_{\mu} .
$$

By the usual theorem of persistence of non-degenerate periodic orbits, there is a unique periodic orbit on $P_{0}=Y$ on each nearby level-set of $H$. This is proved by applying the implicit function 
theorem to the Poincaré return map. Using the same map, but now on $Y \times \mathfrak{g}^{*}$, and restricted to an energy level $H^{-1}(E)$, we again apply the implicit function theorem to eliminate the $Y$-variables, and we are left with a Poisson return map $f_{E}: \mathfrak{g}^{*} \rightarrow \mathfrak{g}^{*}$ (Lemme 1$)$. For each $\mu \in \mathfrak{g}^{*}$, we have, by restriction, a map $f_{E, \mu}: \mathcal{O}_{\mu} \rightarrow \mathcal{O}_{\mu}$, whose fixed points correspond bijectively to the RPOs of energy $E$ and momentum $\mu$. Furthermore, since the map $f_{E, \mu}$ is homotopic to the identity (Lemme 2), and since the Euler characteristic of the coadjoint orbit $\mathcal{O}_{\mu}$ is $w(\mu) \geq 2$, the map $f_{E, \mu}$ has at least one fixed point, and at least $w(\mu)$ if they are all non-degenerate.

\section{Introduction}

Considérons une variété symplectique $(P, \omega)$ et un groupe de Lie $G$ compact et connexe qui agit librement sur $P$ de façon hamiltonienne — c.à.d. il existe une application moment équivariante $\mathbf{J}: P \rightarrow \mathfrak{g}^{*}$, où $\mathfrak{g}$ est l'algèbre de Lie de $G$ et $\mathfrak{g}^{*}$ son duale. Ceci implique que l'application moment soit une submersion, et que l'espace des orbites $P / G$ soit lisse. Pour $\mu \in \mathfrak{g}^{*}$, l'espace réduit associé se définit comme étant

$$
P_{\mu}=\mathbf{J}^{-1}\left(\mathcal{O}_{\mu}\right) / G \subset P / G,
$$

où $\mathcal{O}_{\mu}$ est l'orbite coadjointe de $\mu$ pour l'action de $G$ dans $\mathfrak{g}^{*}$.

Sur $P$ on considère un système hamiltonien défini par une fonction lisse $H: P \rightarrow \mathbf{R}$, qu'on suppose $G$-invariante. Elle induit une fonction sur $P / G$, toujours notée $H$. La dynamique sur $P$ se projette sur un système dynamique de type Poisson-Hamilton sur l'espace des orbites $P / G$, déterminée par le champ de vecteurs de Poisson-Hamilton : $X_{H}=\{H, \cdot\}$ et, ainsi définie, elle préserve les espaces réduits $P_{\mu} \subset P / G$.

Une orbite périodique relative (OPR) dans $P$ est une orbite $\gamma(t)$ telle qu'il existe $g \in G$ et $T>0$ (la période) avec

$$
\gamma(T)=g \cdot \gamma(0)
$$

La projection de $\gamma$ sur $P / G$, encore notée $\gamma$, est évidemment une orbite périodique de période $T$. On supposera que les OPR ne sont pas des équilibres relatifs (c.a.d. G. $\gamma(0)$ n'est pas invariant sous la dynamique). D'après un théorème de Field [2] et Krupa [5], les opr dans $P$ sont des trajectoires qui sont denses sur un tore de dimension au plus $\operatorname{rang}(G)+1$ (ou plus généralement $\operatorname{rang}\left(N_{G}(K)\right)+1$, où $K$ est le sous-groupe d'isotropie des points de $\gamma$ et $N_{G}(K)$ son normalisateur dans $\left.G\right)$.

Dans un système hamiltonien les niveaux d'énergie sont invariants et, si une orbite périodique non-constante sur un niveau d'énergie donné est non-dégénérée, alors cette orbite périodique persiste sur les niveaux d'énergie voisins (l'ensemble des orbites périodiques proches de l'orbite donnée forme un cylindre transverse aux niveaux d'énergie - voir [1] pour un dessin et une démonstration qui consiste en une simple application du théorème des fonctions implicites : le fait que l'orbite soit non-constante fait que les niveaux d'énergie sont réguliers dans un voisinage de l'orbite).

Dans le cas d'un système hamiltonien avec symétrie, on a dans $P / G$ les espaces réduits $P_{\mu}$, qui sont invariants, ainsi que leurs intersections avec les niveaux d'énergie — on parlera des niveaux d'énergie-moment. La question suivante se pose naturellement :

Si une OPR non-constante sur un niveau d'énergie-moment donné est non-dégénérée, persiste-t-elle aux niveaux d'énergie-moment voisins?

Il faut bien-sûr définir la notion de non-dégénérescence pour les OpRs. Mais avant de faire cela, on remarque qu'une complication dans cette situation est que la famille des espaces réduits n'est pas en général une famille régulière (elle n'est pas un feuilletage). Pour que cette famille ne soit pas 


\section{J. Montaldi}

trop 'mauvaise', et aussi pour avoir une réponse affirmative à la question, on suppose que l'action du groupe est libre. On peut construire des exemples où l'action n'est pas localement libre et où l'orbite ne persiste pas. En effet, c'est déjà le cas pour les équilibres relatifs - voir [6].

\section{Persistance}

Une orbite périodique dans un système hamiltonien est dite non-dégénérée si la multiplicité de la valeur propre 1 de l'opérateur de Floquet prend sa valeur minimale, c.à.d. 2. Une opR est dite non-dégénérée si sa projection sur l'espace réduit qui la contient est non-dégénérée dans ce sens. Si en effet l'OPR en question est une vraie orbite périodique dans $P$, alors elle est non-dégénérée si la multiplicité algébrique de 1 comme valeur propre est égale à $2 \operatorname{dim}(G \cdot p)-\operatorname{dim}(G \cdot \mu)+2[9, \S 4(\mathrm{c})]$.

Supposons que l'opR donnée $\gamma$ se trouve dans l'espace réduit $P_{\mu_{*}}$ et qu'on veuille étudier l'existence des OPRs sur les espaces réduits proches. Par un argument dû à Guillemin et Sternberg (et explicité dans [6]), il résulte qu'on peut se ramener au cas $\mu_{*}=0$ : en prenant une tranche à l'action coadjointe en $\mu_{*}$, l'image réciproque $P\left(\mu_{*}\right)$ de cette tranche est une variété symplectique lisse munie d'une action de $G_{\mu_{*}}$ pour laquelle le système hamiltonien induit sur $P\left(\mu_{*}\right) / G_{\mu_{*}}$ est équivalent au sytème hamiltonien sur $P / G$. On suppose dans la suite donc que $\mu_{*}=0$.

Théorème 1 Soit $\gamma$ une OPR du système hamiltonien dans $P_{0}$, non-dégénérée, de période $T_{*}$ et d'énergie $H=E_{*}$. Alors il existe un voisinage $U$ de cette OPR tel que, pour tout $\mu \in \mathfrak{g}^{*}$ suffissament petit et toute énergie $E$ proche de $E_{*}$, il existe un morphisme symplectique $f_{E, \mu}: \mathcal{O}_{\mu} \rightarrow \mathcal{O}_{\mu}$, homotope à l'identité, dont les points fixes sont en bijection avec les OPR dans $P_{\mu} \cap U$, d'énergie $E$ et de période proche de $T_{*}$. En conséquence, il existe au moins une telle OPR et si les OPRs sont non-dégénérées sur $P_{\mu}$ il en existent au moins $w(\mu)$.

Ici $w(\mu)$ est le nombre de Weyl de l'orbite coadjointe $\mathcal{O}_{\mu}[6]$ (égale à son caractéristique d'Euler).

Démonstration Notons $\bar{p}=\gamma(0) \in P_{0}$. Il est bien connu $[10,6]$ que dans un voisinage de $\bar{p}$ l'espace des orbites $P / G$ peut être identifié à un voisinage de l'origine dans le produit d'un espace symplectique $Y$ et de l'espace de Poisson $\mathfrak{g}^{*}$ :

$$
U \stackrel{\sim}{\longrightarrow} Y \times \mathfrak{g}^{*},
$$

où $Y=\operatorname{ker} d \mathbf{J}_{p} / \mathfrak{g} \cdot p$ est la 'tranche symplectique' en $p$. Les espaces réduits s'identifient à

$$
P_{\mu} \cap U \stackrel{\sim}{\longrightarrow} Y \times \mathcal{O}_{\mu} .
$$

On considère la restriction du flot sur $P / G$ à un intervalle de temps $] T_{*}-\varepsilon, T_{*}+\varepsilon[$ et à un voisinage $U$ de $\bar{p}$ :

$$
\phi:] T_{*}-\varepsilon, T_{*}+\varepsilon\left[\times U \rightarrow U^{\prime} .\right.
$$

On prendra les voisinages $U$ et $U^{\prime}$ de (3) assez petits pour que le (2) soit vérifié. La démonstration du théorème se base sur une construction d'un sort de section de Poincaré dont l'application de retour est un morphisme de Poisson de $\mathfrak{g}^{*}$ (Lemme 1), et le fait que cette application de retour est homotope à l'identité (Lemme 2). Les démonstrations des lemmes se trouvent après la démonstration du théorème.

La démonstration habituelle [1] pour la continuation à travers les niveaux d'énergie d'une orbite périodique non-dégénérée consiste en un choix d'une section de Poincaré $\Sigma$ qui détermine une application de retour $\Pi: \Sigma \rightarrow \Sigma$. On décompose $\Sigma=\Sigma\left(E_{*}\right) \times \mathbf{R}$, où $\Sigma\left(E_{*}\right)=\Sigma \cap H^{-1}\left(E_{*}\right)$. La non-dégénérescence de l'orbite périodique nous permet d'utiliser le théorème des fonctions implicites 
pour éliminer les variables dans $\Sigma\left(E_{*}\right)$. Ce qui reste est une application $\mathbf{R} \rightarrow \mathbf{R}$ qui est l'identité à cause de la conservation d'énergie.

Dans notre cas, on modifie à peine cet argument : choisissons une section $\Sigma_{Y}$ dans $Y$ transverse au flot $\phi$ et on pose $\Sigma=\Sigma_{Y} \times \mathfrak{g}^{*}$ - ce qui définit une section transverse au flot dans $Y \times \mathfrak{g}^{*}$. Vu que l'énergie est invariante, on considère les sous-variétés invariantes $\Sigma(E):=\Sigma \cap H^{-1}(E)$ de codimension 2 dans $Y \times \mathfrak{g}^{*}$.

Pour chaque énergie $E$ proche de $E_{*}$, on a dans $Y$ une orbite périodique non-dégénérée d'énergie $E$, notée $\gamma_{E}$, qu'on trouve par la méthode standard (avec $\Sigma=\Sigma_{Y}$ ). L'application de retour restreinte à $\Sigma(E)$ est un morphisme de Poisson qui préserve $Y \cap \Sigma(E)$, par le Lemme 1 .

Lemme 1 Pour chaque énergie $E$ proche de $E_{*}$, l'application de retour $F_{E}: \Sigma(E) \rightarrow \Sigma(E)$ induit un morphisme de Poisson $f_{E}: \mathfrak{g}^{*} \longrightarrow \mathfrak{g}^{*}$, tel que l'ensemble des points fixes de $F_{E}$ est en bijection avec l'ensemble des points fixes de $f_{E}$.

A cause de la conservation du moment, le morphisme $f_{E}$ du lemme préserve les orbites coadjointes $\mathcal{O}_{\mu}$. D'ailleurs, vu que $f_{E}$ est de Poisson, ses restrictions $f_{E, \mu}=f_{\left.E\right|_{\mathcal{O}_{\mu}}}$ sont symplectiques.

Lemme 2 Les applications $f_{E, \mu}$ sont homotope à l'identité.

Un morphisme de $\mathcal{O}_{\mu}$ qui est homotope à l'identité a au moins un point fixe, car son nombre de Lefschetz est égale à la caractéristique d'Euler, qui pour une telle variété est différente de zéro (en effet, les $H^{r}\left(\mathcal{O}_{\mu}\right)$ sont nuls pour $r$ impair). Dans certains cas, le fait que $f_{E, \mu}$ soit symplectique entraine qu'il y a plus de points fixes.

Démonstration du Lemme 1 Par la non-dégénérescence de l'orbite $\gamma_{E}$ on peut éliminer les variables dans $Y \cap \Sigma(E)$ (par le théorème des fonctions implicites comme dans la démonstration standard) et il nous reste donc, pour chaque $E$, un morphisme $f_{E}: \mathfrak{g}^{*} \rightarrow \mathfrak{g}^{*}$ dont on montre qu'il est de Poisson grace au Lemme 3.

Lemme 3 Soient $U, V$ deux espaces symplectiques et $g: U \oplus V \rightarrow U, h: U \oplus V \rightarrow V$ telles que $F=g \oplus h: U \oplus V \rightarrow U \oplus V$ soit un morphisme symplectique. Soit $0 \in U$ un point fixe nondégénéré de $h_{0}: V \rightarrow V, v \mapsto h(0, v)$. Par le théorème des fonctions implicites, pour chaque u assez petit, il existe $v=\lambda(u)$ telle que $h(u, \lambda(u))=\lambda(u)$. Alors, l'application $f: U \rightarrow U$ définie par $f(u)=g(u, \lambda(u))$ est un morphisme symplectique. Le résultat analogue pour les variétés de Poisson est aussi vrai.

Démonstration On a $\omega_{U}+\omega_{V}=F^{*}\left(\omega_{U}+\omega_{V}\right)=g^{*} \omega_{U}+h^{*} \omega_{V}$. L'image réciproque par rapport à $\Gamma=\left(1_{U}, \lambda\right): U \rightarrow U \oplus V$ donne le résultat, vu que : $\Gamma^{*} \omega_{U}=\omega_{U}, \Gamma^{*} \omega_{V}=\lambda^{*} \omega_{V}, f=g \circ \Gamma$ et $\lambda=h \circ \Gamma$. Pour les variétés de Poisson, on remarque que si une application est symplectique sur toutes les feuilles symplectiques, alors elle est de Poisson.

Démonstration du Lemme 2 Considérons le flot en temps $T$ en $P, \Phi_{T}: P \rightarrow P$. Vu que $\Phi_{T}(p)=g \cdot p$, on peut définir une application de retour $\sigma:=g^{-1} \circ \Phi_{T}$ sur $P$ qui vérifie $\sigma(p)=p$. Par rapport à la décomposition locale $P \stackrel{\sim}{\longrightarrow} G \times \mathfrak{g}^{*} \times Y$, la dérivée de $\sigma$ est de la forme,

$$
d \sigma(p)=\left[\begin{array}{ccc}
A d_{g^{-1}} & * & * \\
0 & A d_{g^{-1}}^{*} & 0 \\
0 & * & \sigma_{Y}
\end{array}\right]
$$

où les $*$ s designent des sous-matrices indéterminées. La premiére colonne est un calcul simple : $\sigma(h . p)=g^{-1} \phi_{T}(h . p)=g^{-1} h \cdot \phi_{T}(p)=\left(g^{-1} h g\right) \cdot \sigma(p)=\left(g^{-1} h g\right) \cdot p ;$ la deuxième ligne découle de la conservation du moment, car l'application moment est de la forme $\mathbf{J}(g, \mu, y)=A d_{g^{-1}}^{*} \mu$. 
La projection de $\sigma$ sur l'espace des orbites est $\phi_{T}$, et donc

$$
d \phi_{T}(p)=\left[\begin{array}{cc}
A d_{g^{-1}}^{*} & 0 \\
* & \sigma_{Y}
\end{array}\right] .
$$

Après avoir éliminé $Y$, il nous reste

$$
d f_{E_{*}}(p)=A d_{g^{-1}}^{*},
$$

qui est homotope à l'identité, car $G$ est connexe. L'application $f_{E, \mu}$ est une perturbation de la restriction de $A d_{g^{-1}}^{*}$ à $\mathcal{O}_{\mu}$ (on ajoute la partie non-linéaire de $f_{E_{*}}$ et on change le niveau d'énergie de façon continue), et donc elle aussi est homotope à l'identité.

\section{Une application}

Récemment, Stewart [11] a étudié le problème de $N$-corps identiques, avec une hypothèse de hamiltonien non-singulier (les corps peuvent passer l'un à travers l'autre). Pour le cas $N=4$, il a trouvé des solutions de mouvement radial qui sont des orbites périodiques de moment cinétique nul. Le groupe de symétries de ce problème est $\mathbf{S O}(3)$ (qui agit librement), fois $S_{4}$ (le groupe de permutations des 4 corps) i.e. $\mathbf{S O}(3) \times S_{4}$. Ces orbites ont une isotropie égale au groupe $S_{4}$. On trouve le même type de solution comme mouvement vibratoire d'une molécule de méthane $\mathrm{CH}_{4}$ (voir [8]). On peut supposer que ces orbites périodiques sont non-dégénérées. Alors selon le théorème 1 , pour chaque valeur (petite) du moment cinétique, il existe une application symplectique $f_{\mu}: \mathcal{O}_{\mu} \rightarrow \mathcal{O}_{\mu}$ (dans ce cas $\mathcal{O}_{\mu} \simeq S^{2}$ pour $\mu \neq 0$ ) qui commute à l'action coadjointe de $S_{4}$, dont les points fixes correspondent aux OPRs voisines.

Il se trouve [7] que l'action coadjointe de $S_{4}$ a 24 points fixes, et donc $f_{E, \mu}$ a au moins 24 points fixes, ce qui donne 24 oprs pour chaque $E, \mu$. L'action de $S_{4}$ identifie certaines de ces oprs, et en effet il y en a trois types différents : ceux fixés par les rotations d'ordre 3, ceux par des éléments d'ordre 4, et ceux par des rotations d'ordre 2. On rappel que si un symplectomorphisme de $S^{2}$ a plus que 2 points fixes, il a un nombre infinie de points périodiques $[4,3]$, et donc dans ce problème il y a un nombre infine d'oprs pour chaque $E, \mu$, mais de période arbitrairement long.

\section{Références bibliographiques}

[1] Abraham R. et Marsden J., 1978. Foundations of Mechanics. Addison-Wesley.

[2] Field M., 1980. Equivariant dynamical systems. Trans. A.M.S. 259 pp. 185-205.

[3] Franks J., 1992. Geodesics on $S^{2}$ and periodic points of annulus homeomorphisms. Invent. Math. 108 pp. $403-418$.

[4] Gambaudo J.-M., et Le Calvez P., 1991. Infinité d'orbites périodiques pour les difféomorphismes de l'anneau. Preprint.

[5] Krupa M., 1990. Bifurcations of relative equilibria. SIAM J. Math. Anal. 21 pp. 1453-1486.

[6] Montaldi J., 1997. Persistence and stability of relative equilibria. Nonlinearity 10 pp. $449-466$.

[7] Montaldi J., et Roberts R.M., 1997. Relative equilibria of molecules. Preprint.

[8] Montaldi J., Roberts R.M., et Stewart I., 1987. Nonlinear normal modes of symmetric Hamiltonian systems. In Pattern Formation in Physics (Güttinger W., Dangelmayr G., Ed), Springer-Verlag.

[9] Montaldi J., Roberts R.M., et Stewart I., 1988. Periodic solutions near equilibria of symmetric Hamiltonian systems. Phil. Trans. Roy. Soc. London 325, pp. $237-293$.

[10] Sjamaar R. et Lerman E., 1991. Stratified symplectic spaces and reduction. Annals of Math. 134, pp. 375-422.

[11] Stewart I., 1996. Symmetry methods in collisionless many-body problems. Journal of Nonlinear Science 6, pp. 543 $-563$. 